\title{
Ensemble-Based Modeling of the NMR Spectra of Solid Solutions: Cation Disorder in $\mathrm{Y}_{2}(\mathrm{Sn}, \mathrm{Ti})_{2} \mathrm{O}_{7}$
}

\author{
Robert F. Moran, ${ }^{\dagger}$ David McKay, ${ }^{\dagger}$ Paulynne C. Tornstrom, ${ }^{\ddagger}$ Alex Aziz, ${ }^{\ddagger}$ Arantxa Fernandes, ${ }^{\dagger}$

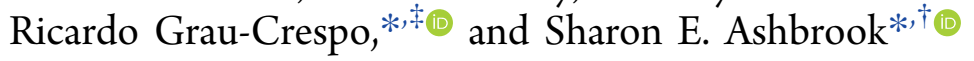 \\ ${ }^{\dagger}$ School of Chemistry, EaStCHEM and Centre of Magnetic Resonance, University of St Andrews, St Andrews KY16 9ST, U.K. \\ ${ }^{\ddagger}$ Department of Chemistry, University of Reading, Whiteknights RG6 6AD, U.K.
}

\section{Supporting Information}

\begin{abstract}
The sensitivity of NMR to the local environment, without the need for any long-range order, makes it an ideal tool for the characterization of disordered materials. Computational prediction of NMR parameters can be of considerable help in the interpretation and assignment of NMR spectra of solids, but the statistical representation of all possible chemical environments for a solid solution is challenging. Here, we illustrate the use of a symmetry-adapted configurational ensemble in the simulation of NMR spectra, in combination with solid-state NMR experiments. We

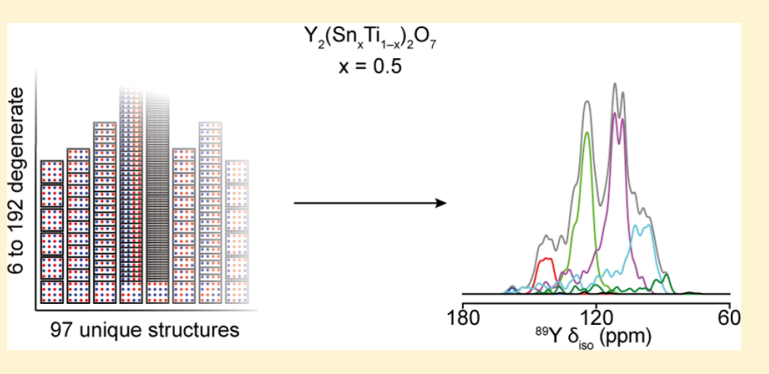
show that for interpretation of the complex and overlapped lineshapes that are typically observed, it is important to go beyond a single-configuration representation or a simple enumeration of local environments. The ensemble method leads to excellent agreement between simulated and experimental spectra for $\mathrm{Y}_{2}(\mathrm{Sn}, \mathrm{Ti})_{2} \mathrm{O}_{7}$ pyrochlore ceramics, where the overlap of signals from different local environments prevents a simple decomposition of the experimental spectral lineshapes. The inclusion of a Boltzmann weighting confirms that the best agreement with experiment is obtained at higher temperatures, in the limit of full disorder. We also show that to improve agreement with experiment, in particular at low dopant concentrations, larger supercells are needed, which might require alternative simulation approaches as the complexity of the system increases. It is clear that ensemble-based modeling approaches in conjunction with NMR spectroscopy offer great potential for understanding configurational disorder, ultimately aiding the future design of functional materials.
\end{abstract}

\section{INTRODUCTION}

The mixing of different elements over specified sites within a crystal lattice, forming a solid solution, offers a powerful and commonly exploited route to engineer the properties of materials. For example, band gap engineering via compositional optimization has been used for several decades to obtain the desired optoelectronic behavior in semiconducting alloys. ${ }^{1}$ However, the detailed structural characterization of such disordered materials, vital for understanding structureproperty relationships, can be extremely challenging, with methods based on Bragg diffraction providing a picture of the structure that is averaged over space and time. While this average picture can be useful in understanding or predicting some selected properties of materials, detailed information on the local atomic environment of individual atoms, which may play an important role in chemical reactivity, is often lost. Nuclear magnetic resonance (NMR) spectroscopy provides a complementary tool for the study of disordered or dynamic systems, with its inherent sensitivity to the atomic-scale geometry through interactions such as the chemical shift. ${ }^{2,3}$ However, the complexity of the spectral lineshapes obtained for disordered systems hinders the straightforward extraction of structural information, and attention has turned to computation to assist spectral interpretation. ${ }^{4,5}$ In most cases, this has involved the use of simple substitutions into an ordered parent structure to gain insight into the typical type and magnitude of the changes that are seen in the NMR interaction of interest. ${ }^{4,5}$ Although this may facilitate a better understanding of the likely effect of disorder on the NMR spectrum, it falls far short of providing the detailed structural picture that is usually desired.

In general, computer simulation is a useful tool for the design of functional solid solutions. However, the computational modeling of site-disordered solids is more challenging owing to configurational complexity, and periodic boundary conditions often cannot be applied directly as in the case of ordered crystals. ${ }^{6}$ The theoretical investigation of the thermodynamics of disorder and mixing in solid solutions typically involves evaluating the energies of structures within a large configurational space, which becomes affordable by using an effective Hamiltonian that gives the energy as a function of site occupancies, for example using a cluster expansion approach. $^{7-9}$ The effective interactions are parametrized using density functional theory (DFT) calculations or experimental information. Large supercells and many config-

Received: August 21, 2019

Published: October 8, 2019 
urations are normally required to achieve convergence of the configurational entropy and the free energy of the solid solutions. In contrast, when other properties of the solid solution (i.e., those beyond thermodynamics such as electronic behavior) are to be evaluated, the most common approach is to use a single configuration that is representative of the mixed system. For highly ordered solid solutions, the lowest-energy configuration is chosen, whereas for highly disordered systems, a good representative configuration is considered to be the special quasi-random structure (SQS), which mimics the pair correlation function of the fully disordered solid. ${ }^{10}$

In this work we show that for the prediction of NMR parameters, and interpretation of the complex and overlapped lineshapes that are typically observed in NMR spectra of disordered solids, it is important to go beyond a singleconfiguration representation and employ configurational ensembles. Such an approach allows the systematic enumeration of all possible chemical environments around a given cation but, unlike the simple "cluster-based" models applied previously, ${ }^{4,5}$ includes proper consideration of the statistical weights including configurational degeneracy and energetic preference effects. As the evaluation of NMR chemical shieldings is computationally much more expensive than a simple energy evaluation (especially when a cluster expansion is used for the latter), the ensembles are necessarily limited to a cell (or supercell) smaller than those typically used for thermodynamic calculations. However, if the cell is sufficiently large to include the number of neighbors affecting the contributions to the NMR interactions of interest, a robust prediction of the complex NMR spectra can be obtained. Here, we demonstrate this using the $\mathrm{Y}_{2}(\mathrm{Sn}, \mathrm{Ti})_{2} \mathrm{O}_{7}$ pyrochlore system (studied previously using ${ }^{89} \mathrm{Y},{ }^{119} \mathrm{Sn}$ and ${ }^{17} \mathrm{O}$ NMR spectroscopy $)^{11-17}$ as a case study. We show that these calculations allow a clear interpretation of the origin of the features in the NMR spectra of a solid solution, and provide insight into the atomic configurations that are present in the real material, and the importance each has in determining the appearance of the solid-state NMR spectrum. We demonstrate that the combination of NMR spectroscopy and quantum-mechanical simulations that consider configurational probabilities can offer crucial insights into the short-range effects and ordering in solid solutions, which escape most diffraction-based measurements.

\section{METHODS}

Synthesis and Basic Characterization. $\mathrm{Y}_{2}\left(\mathrm{Sn}_{x} \mathrm{Ti}_{1-x}\right)_{2} \mathrm{O}_{7}(x=$ 0.0 to 1.0 in steps of 0.125 ) samples were synthesized (in a similar manner to previous work) using a conventional solid-state mixed metal oxide process. Stoichiometric quantities of commercially available $\mathrm{Y}_{2} \mathrm{O}_{3}$ (Sigma-Aldrich, 99\%), $\mathrm{SnO}_{2}$ (Sigma-Aldrich, 99.9\%), and $\mathrm{TiO}_{2}$ (Sigma-Aldrich, 99\%) were ball milled $(1 \mathrm{~h})$ using $\mathrm{ZrO}_{2}$ balls and acetone/cyclohexanol as the milling medium. The dry powders were then pressed into a pellet, placed in an alumina boat, and heated to $\sim 1673 \mathrm{~K}$ (at $5 \mathrm{~K} \mathrm{~min}^{-1}$ ) for $48 \mathrm{~h}$. The pellets were reground and repressed before a second sintering at $\sim 1673 \mathrm{~K}$ for a further $48 \mathrm{~h}$. After cooling (also at $5 \mathrm{~K} \mathrm{~min}^{-1}$ ), the samples were assessed for phase purity using a PANalytical Empyrean diffractometer, $\mathrm{Cu} \mathrm{K} \mathrm{K}_{\alpha 1}$ radiation $(\lambda=1.5406 \AA)$, and an $\mathrm{X}^{\prime}$ celerator linear detector. Patterns were collected over a $2 \theta$ angular range of $10-100^{\circ}$ with a step size of $0.02^{\circ}$ and a step duration of $0.4 \mathrm{~s}$. Samples were shown to be single-phase pyrochlore throughout (see Supporting Information).

Solid-State NMR Spectroscopy. ${ }^{89} \mathrm{Y}$ NMR spectra were acquired using a Bruker Avance III $600 \mathrm{MHz}$ NMR spectrometer, equipped with a widebore $14.1 \mathrm{~T}$ magnet, at a Larmor frequency of
29.41 MHz. Powdered samples were packed into $4 \mathrm{~mm} \mathrm{Si}_{3} \mathrm{~N}_{4}$ rotors to prevent any ${ }^{89} \mathrm{Y}$ background signal and rotated at $14 \mathrm{kHz}$, using a 4 mm HX low- $\gamma$ probe. Spectra were acquired using a spin-echo pulse sequence, with a radiofrequency field strength of $\sim 22 \mathrm{kHz}(\pi / 2 \approx$ $11.3 \mu \mathrm{s})$ and a recycle interval of $30 \mathrm{~s}$. Although $T_{1}$ is relatively long for all ${ }^{89} \mathrm{Y}$ resonances, there is little difference in the relative relaxation rates, and spectral intensities accurately reflect the relative site populations even at shorter recycle intervals. ${ }^{13}$ Chemical shifts are given in ppm relative to the primary reference $1 \mathrm{M}$ aqueous $\mathrm{YCl}_{3}$, measured using a secondary reference compound, $\mathrm{Y}_{2} \mathrm{Ti}_{2} \mathrm{O}_{7}$, at 65 ppm. $^{12}$

${ }^{119} \mathrm{Sn}$ NMR spectra were acquired using a Bruker Avance III 400 $\mathrm{MHz}$ spectrometer, equipped with a widebore $9.4 \mathrm{~T}$ magnet, at a Larmor frequency of $149.2 \mathrm{MHz}$. Powdered samples were packed into $4 \mathrm{~mm} \mathrm{ZrO}_{2}$ rotors and rotated at $14 \mathrm{kHz}$, using a $4 \mathrm{~mm} \mathrm{HX}$ probe. Spectra were acquired using a spin-echo, with a radiofrequency field strength of $\sim 111 \mathrm{kHz}(\pi / 2 \approx 2.25 \mu \mathrm{s})$ and a recycle interval of $30 \mathrm{~s}$. Chemical shifts are given in $\mathrm{ppm}$ relative to the primary reference $\left(\mathrm{CH}_{3}\right)_{4} \mathrm{Sn}$, measured using a secondary reference compound, $\mathrm{SnO}_{2}$, at $-604.3 \mathrm{ppm}$.

Calculations. The Site Occupancy Disorder (SOD) program $^{18}$ was used to generate the set of symmetrically inequivalent configurations for each number $k$ of $\mathrm{B}$-site substitutions in a pyrochlore unit cell $\mathrm{Y}_{16} \mathrm{~B}_{16-k} \mathrm{~B}_{k}{ }_{k} \mathrm{O}_{56}$. Two configurations are equivalent if there is an isometric transformation that converts one into the other. The transformations considered are simply the symmetry operators of the parent structure (in this case those in the $F d \overline{3} m$ space group of the cubic pyrochlore structure). The total number of atomic configurations $(W)$ and the number of inequivalent configurations $(M)$ for each pyrochlore composition are listed in

Table 1. Total Number of Atomic Configurations $(W)$ and the Number of Symmetry Inequivalent Configurations $(M)$ for $\mathrm{Y}_{2}\left(\mathrm{Sn}_{x} \mathrm{Ti}_{1-x}\right)_{2} \mathrm{O}_{7}$ Structures in a Single Cubic Unit Cell (with 16 Exchangeable B Sites) Generated Using SOD

\begin{tabular}{lllll}
\multicolumn{1}{c}{ chemical formula } & \multicolumn{1}{c}{$x$} & B site NNN & \multicolumn{1}{c}{$W$} & $M$ \\
$\mathrm{Y}_{2} \mathrm{Sn}_{2} \mathrm{O}_{7}$ & 1.0 & Sn16 & 1 & 1 \\
$\mathrm{Y}_{2}\left(\mathrm{Sn}_{0.875} \mathrm{Ti}_{0.125}\right)_{2} \mathrm{O}_{7}$ & 0.875 & Sn14Ti2 & 120 & 3 \\
$\mathrm{Y}_{2}\left(\mathrm{Sn}_{0.75} \mathrm{Ti}_{0.25}\right)_{2} \mathrm{O}_{7}$ & 0.75 & Sn12Ti4 & 1820 & 22 \\
$\mathrm{Y}_{2}\left(\mathrm{Sn}_{0.625} \mathrm{Ti}_{0.375}\right)_{2} \mathrm{O}_{7}$ & 0.625 & Sn10Ti6 & 8008 & 65 \\
$\mathrm{Y}_{2}\left(\mathrm{Sn}_{0.5} \mathrm{Ti}_{0.5}\right)_{2} \mathrm{O}_{7}$ & 0.5 & Sn8Ti8 & 12870 & 97 \\
$\mathrm{Y}_{2}\left(\mathrm{Sn}_{0.375} \mathrm{Ti}_{0.625}\right)_{2} \mathrm{O}_{7}$ & 0.375 & Sn6Ti10 & 8008 & 65 \\
$\mathrm{Y}_{2}\left(\mathrm{Sn}_{0.25} \mathrm{Ti}_{0.75}\right)_{2} \mathrm{O}_{7}$ & 0.25 & Sn4Ti12 & 1820 & 22 \\
$\mathrm{Y}_{2}\left(\mathrm{Sn}_{0.125} \mathrm{Ti}_{0.875}\right)_{2} \mathrm{O}_{7}$ & 0.125 & Sn2Ti14 & 120 & 3 \\
$\mathrm{Y}_{2} \mathrm{Ti}_{2} \mathrm{O}_{7}$ & 0.0 & Ti16 & 1 & 1 \\
total & & & 32768 & 279 \\
\hline
\end{tabular}

Table 1. The total number of configurations is given by simple statistics as

$$
W=N ! /((N \times x) !(N \times(1-x)) !)
$$

where $N$ is the number of sites over which substitutions are considered (i.e., 16 for the $\mathrm{B}$ sites in the pyrochlore structure) and $x=$ $k / N$ is the molar fraction of substitutions. The geometry of each of the 279 resulting unique structural models was then optimized using the CASTEP DFT code (version 8.0). ${ }^{19,20}$ DFT calculations were performed using the $\mathrm{PBE}^{21}$ exchange-correlation functional, and corevalence interactions were described by ultrasoft pseudopotentials, ${ }^{22}$ accounting for scalar relativistic effects using ZORA. ${ }^{23}$ A planewave energy cutoff of $60 \mathrm{Ry}(\sim 816 \mathrm{eV})$ was used, with the first Brillouin zone sampled through a Monkhorst-Pack grid $^{24}$ with a reciprocal space grid spacing of $0.042 \pi \AA^{-1}$. In the geometry optimization all atomic coordinates and unit cell parameters were allowed to vary, with a geometry optimization energy tolerance of $1 \times 10^{-5} \mathrm{eV}$ per atom and an electronic structure energy tolerance of $1 \times 10^{-9} \mathrm{eV}$ per atom used (see Supporting Information). From these energies, 

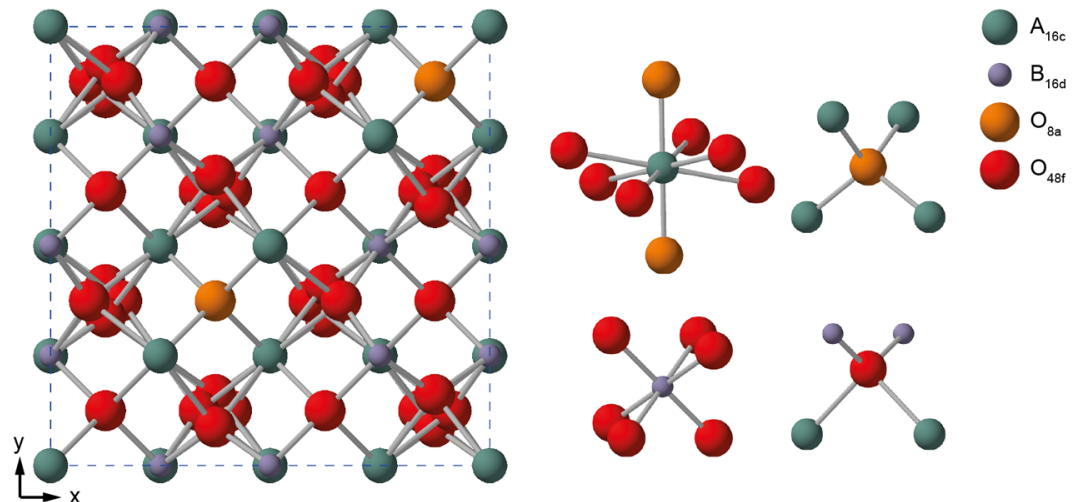

b

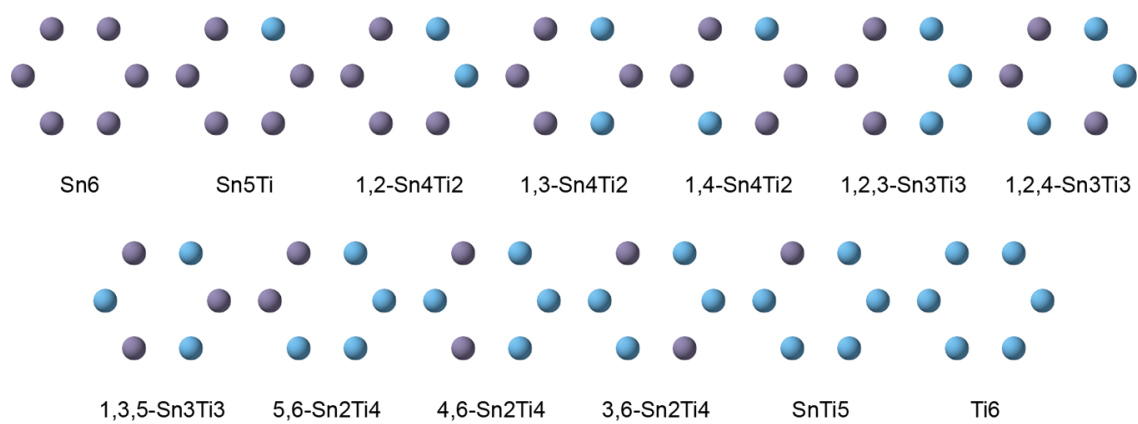

Figure 1. (a) Structure of a typical $\mathrm{A}_{2} \mathrm{~B}_{2} \mathrm{O}_{7}$ pyrochlore, with expansions of the local environments of the Wykoff $16 \mathrm{c}(\mathrm{A}), 16 \mathrm{~d}(\mathrm{~B})$, and $8 \mathrm{a}$ and $48 \mathrm{f}$ (O) sites. (b) Possible arrangements of Sn and Ti on the six NNN B sites that surround the pyrochlore A and B sites. See the Supporting Information for the numbering scheme used.

thermodynamic properties (enthalpies of mixing, $\Delta H_{\text {mix }}$ and free energies of mixing, $\Delta G_{\text {mix }}$ ) were derived in the limit of full disorder and fitted to an asymmetric function using the subregular solid solution model, ${ }^{25}$ as described in the Supporting Information.

NMR parameters were calculated with CASTEP (version 8.0), using the gauge-including projector augmented wave (GIPAW) approach $^{21,22}$ to reconstruct the all-electron wave function in the presence of a magnetic field, and the same parameters as in the geometry optimizations. Calculations generate the absolute shielding tensor $\left(\boldsymbol{\sigma}^{\text {calc }}\right)$ in the crystal frame, and diagonalization of the symmetric part yields the three principal components $\sigma_{11}^{\text {calc }}, \sigma_{22}$ calc, and $\sigma_{33}{ }^{\text {calc }}$. From these, $\sigma_{\text {iso }}{ }^{\text {calc }}=\left(\sigma_{11}^{\text {calc }}+\sigma_{22}{ }^{\chi \alpha \lambda \chi}+\sigma_{33}{ }^{\chi \alpha \lambda \lambda}\right) / 3$, the magnitude of the anisotropy or span, $\Omega^{\chi \alpha \lambda \chi}=\sigma_{11}{ }^{\text {calc }}-\sigma_{33}$ calc, and the skew, $\kappa^{\text {calc }}=3\left(\sigma_{22}{ }^{\chi \alpha \lambda \chi}-\sigma_{\text {iso }}{ }^{\text {calc }}\right) / \Omega^{\text {calc }}$ can be determined. To facilitate comparison to experiment, the corresponding isotropic chemical shift, $\delta_{\text {iso }}{ }^{\text {calc }}$, was determined from $\sigma_{\text {iso }}{ }^{\text {calc }}$. See Supporting Information for further details of the referencing methods used in this work. Predicted NMR spectra were simulated for each structural model by representing each value of $\delta_{\text {iso }}$ calc by an individual Gaussian function (with a fixed line broadening of $1 \mathrm{ppm}$ ). These spectra were weighted by the corresponding configurational degeneracy of the structural model, before being summed to produce a final spectrum for each composition. In some cases, the spectral intensities were also weighted by the Boltzmann distribution (see Section S2 in the Supporting Information).

\section{RESULTS AND DISCUSSION}

The $\mathrm{A}_{2} \mathrm{~B}_{2} \mathrm{O}_{7}$ pyrochlore structure is based on a supercell of fluorite $\left(\mathrm{AO}_{2}\right)$ with one-eighth of the anions removed in an ordered manner, giving an eight-coordinate A site (occupied here by $\mathrm{Y}^{3+}$ ) and a six-coordinate B site (occupied here by $\mathrm{Sn}^{4+}$ and/or $\left.\mathrm{Ti}^{4+}\right)$, as shown in Figure $1 .^{26-28}$ For each $\mathrm{Y}_{2}\left(\mathrm{Sn}_{x} \mathrm{Ti}_{1-x}\right)_{2} \mathrm{O}_{7}$ composition $(x=0.0$ to 1.0 in steps of $0.125)$ the set of unique structural models was determined as described above using SOD. As described in the Supporting Information, the thermodynamics of mixing in $\mathrm{Y}_{2}(\mathrm{Sn}, \mathrm{Ti})_{2} \mathrm{O}_{7}$ were investigated using the subregular solid solution model as a first approximation, ${ }^{25}$ where a finite enthalpy of mixing, $\Delta H_{\text {mix }}$, is determined (and fitted to an asymmetric function, as explained below), but where the entropy of mixing is assumed to be that of the ideal solution, thus eliminating any complications related to the convergence of the calculated entropy with the size of the simulation cell.

Figure 2a shows a plot of $\Delta H_{\text {mix }}$ for each composition in the limit of full disorder (i.e., the average of the configuration energies weighted only by their corresponding degeneracies).

Values are plotted for each structural model separately in the Supporting Information. The values in Figure $2 \mathrm{a}$ can be fitted to obtain an analytical expression using a second-order Guggenheim polynomial;

$$
\Delta H_{\text {mix }}=x(1-x)\left[W_{0}+W_{1}(2 x-1)\right]
$$

giving $W_{0}=8.88 \mathrm{~kJ} \mathrm{~mol}^{-1}$ and $W_{1}=5.42 \mathrm{~kJ} \mathrm{~mol}^{-1}$. In principle, a better fitting can be achieved using higher-order Guggenheim polynomials, but the improved precision is likely to be irrelevant within the accuracy of the calculations. The enthalpy of mixing is positive and, interestingly, is asymmetric, indicating it is generally easier to substitute $\mathrm{Sn}$ into $\mathrm{Y}_{2} \mathrm{Ti}_{2} \mathrm{O}_{7}$ than to substitute $\mathrm{Ti}$ in $\mathrm{Y}_{2} \mathrm{Sn}_{2} \mathrm{O}_{7}$. This is somewhat surprising, as the ionic radius of $\mathrm{Sn}^{4+}(0.69 \AA)$ is greater than that of $\mathrm{Ti}^{4+}$ $(0.61 \AA),{ }^{29}$ and it is often more difficult to substitute a larger ion in a smaller ion site than vice versa.

Figure $2 \mathrm{~b}$ plots the free energy of mixing $\left(\Delta G_{\text {mix }}\right)$ as a function of temperature. At high temperatures (e.g., $1000 \mathrm{~K}$ ), the entropic term dominates and the solid solution is predicted to be stable with respect to phase separation. However, at 


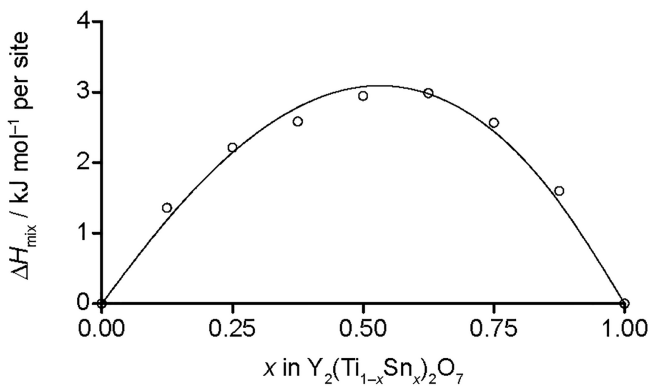

b

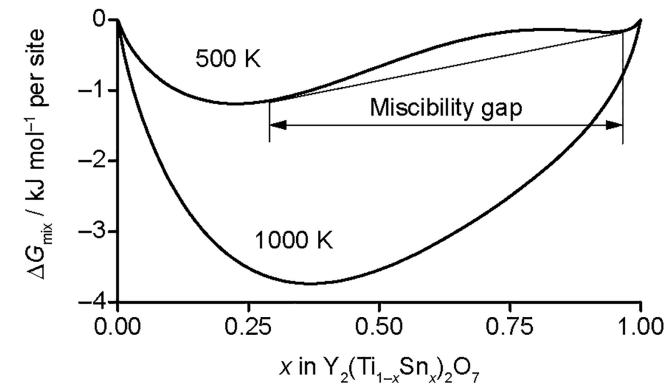

C

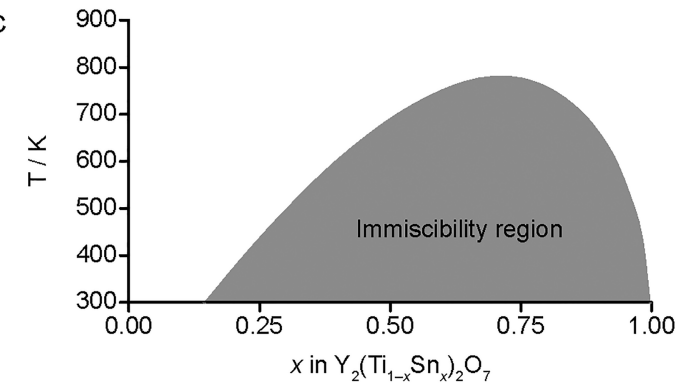

Figure 2. (a) Enthalpy of mixing (from DFT calculations) in the limit of full disorder (circles) and Guggenheim polynomial fitting using a subregular model (line) for $\mathrm{Y}_{2}\left(\mathrm{Sn}_{x} \mathrm{Ti}_{1-x}\right)_{2} \mathrm{O}_{7}$. (b) Free energy of mixing in the subregular model at $500 \mathrm{~K}$ (showing the miscibility gap) and at $1000 \mathrm{~K}$. (c) Phase diagram showing the immiscibility regions where the solid solution is predicted to be metastable with respect to phase separation using the subregular solid solution model.

lower temperatures a miscibility gap appears. This corresponds approximately to the region of composition between the two minima $x_{1}$ and $x_{2}$ of $\Delta G_{\text {mix }}$; that is, a physical mixture of two solid solutions with compositions $x_{1}$ and $x_{2}$ has lower free energy than the solid solution with composition $x$ such that $x_{1}$ $<x<x_{2}$. Due to the asymmetry, the limits of the miscibility gap do not correspond exactly to the minima of $G_{\text {mix }}(x)$, but to points of intersection with the secant, as shown in Figure $2 b$. From this, it is possible to create a phase diagram showing the predicted regions of composition/temperature stability of the solid solution (Figure 2c). This shows a significant immiscibility region is predicted below $800 \mathrm{~K}$, where intermediate compositions are expected to be unstable with respect to decomposition into Ti-rich and Sn-rich solids. Experimentally, however, such compositions do exist as solid solutions $^{13,14}$ (see below), but they are metastable. It should be noted that this analysis is limited by the assumption of the subregular solid solution model, which is expected to overestimate the configurational entropy by assuming ideal mixing. The real system is expected to have lower configurational entropy, and a slightly larger region of immiscibility would be predicted. We have also ignored finite-temperature effects arising from vibrational contributions to the thermodynamics of mixing, which are typically much smaller than configurational effects. ${ }^{30,31}$ This simplifies the calculations considerably, as we can use the $0 \mathrm{~K}$ energies from DFT for the statistical analysis at finite temperature, but this is not expected to substantially affect the results.

For a specified $\mathrm{Y}_{2}\left(\mathrm{Sn}_{x} \mathrm{Ti}_{1-x}\right)_{2} \mathrm{O}_{7}$ composition, it is possible to predict the corresponding ${ }^{89} \mathrm{Y}$ and ${ }^{119} \mathrm{Sn}(I=1 / 2)$ NMR spectra in the limit of full disorder by summing the complete set of spectra predicted for each structural model (after weighting each by the corresponding configurational degeneracy; see above). These predicted spectra are shown in Figure 3, alongside the corresponding experimental spectra. Although ${ }^{89} \mathrm{Y}$ and ${ }^{119} \mathrm{Sn}$ NMR spectra of $\mathrm{Y}_{2}\left(\mathrm{Sn}_{x} \mathrm{Ti}_{1-x}\right){ }_{2} \mathrm{O}_{7}$ have been published previously, ${ }^{13-15}$ Figure 3 shows new experimental spectra for solid solution compositions that match those used in the calculations. ${ }^{32}$ It is clear in all cases (and from the powder XRD measurements discussed above) that a pyrochlore solid solution is formed experimentally, rather than the phase separation predicted by the thermodynamic analysis at room temperature, likely reflecting a metastable freezing of the high-temperature disordered distribution, given the high synthesis temperature $(\sim 1673 \mathrm{~K})$. For both nuclei, surprisingly good agreement is seen between the experimental and predicted NMR spectra, with the latter matching well the number and position of the resonances observed in each case. For the calculated spectra, it is possible to determine the contributions from species with different local environments. Variation of the atoms on the six next nearest neighbor (NNN) B sites surrounding the A and B sites produces 13 different local coordination geometries, which are shown schematically (along with the numbering schemes used) in Figure 1c and the Supporting Information.

Figure $4 \mathrm{a}$ and $\mathrm{d}$ show simulated ${ }^{89} \mathrm{Y}$ and ${ }^{119} \mathrm{Sn}$ MAS NMR spectra for $\mathrm{Y}_{2} \mathrm{SnTiO}_{7}$ (i.e., $x=0.5$ ), decomposed to show the contributions from species with differing numbers, $n$, of $S n$ NNN. (Similar decompositions are shown for all compositions in the Supporting Information). For ${ }^{89} \mathrm{Y}$ (Figure 4a), there is a systematic decrease in the isotropic chemical shift as $n$ decreases, with resolved signals resulting from contributions primarily from $\mathrm{Y}$ species with different $n$. Different arrangements of $\mathrm{B}$ site cations (i.e., environments with the same $n$, but different positions of the $\mathrm{Sn} / \mathrm{Ti} \mathrm{NNN}$, show typically smaller differences in chemical shift. However, as shown in Figure $4 b$, these smaller differences account for the pronounced splitting in the resonance at $\sim 100 \mathrm{ppm}$ (shown expanded in the inset) that was observed, but not explained, in previous work. ${ }^{13,14}$ It is now clear that this splitting results from the different shifts seen for the 1,2,4-Sn3Ti3 environment ( $\sim 5$ ppm higher than for 1,2,3-Sn3Ti3 and 1,3,5-Sn3Ti3 environments). However, it can be seen in Figure $4 \mathrm{c}$ (where $\delta_{\text {iso }}^{\text {calc }}$ values for Y species in all structural models are plotted) and in the spectra shown in the Supporting Information that in most cases there is considerable overlap of the ranges of chemical shift seen for different environments. Therefore, although it may be possible to decompose spectra (and consider integrated intensities of individual resonances, as in previous work ${ }^{13,14}$ ) at low $\mathrm{Sn}$ and $\mathrm{Ti}$ concentrations, for materials with higher levels of disorder this is simply not accurate, with the spectral intensity at any point arising from contributions from species with different $n$. Figure $4 \mathrm{c}$ also shows that as the number of Ti NNN increases (i.e., $n$ decreases), there is significantly more variation in $\delta_{\text {iso }}{ }^{\text {calc }}$ for a particular local environment, leading to greater overlap of 
a

$x=1$
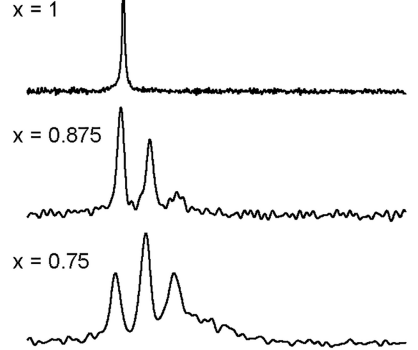

$x=0.625 \mathrm{~N} / \mathrm{N}$

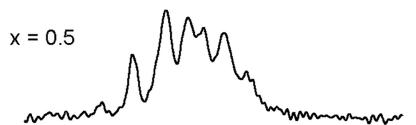

$x=0.375$

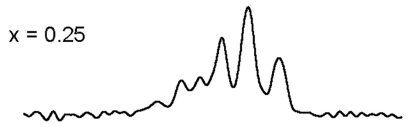

$x=0.125$

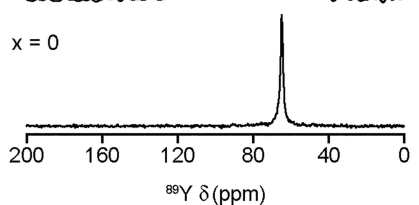

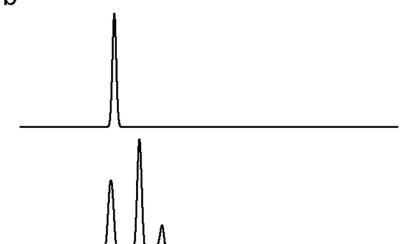
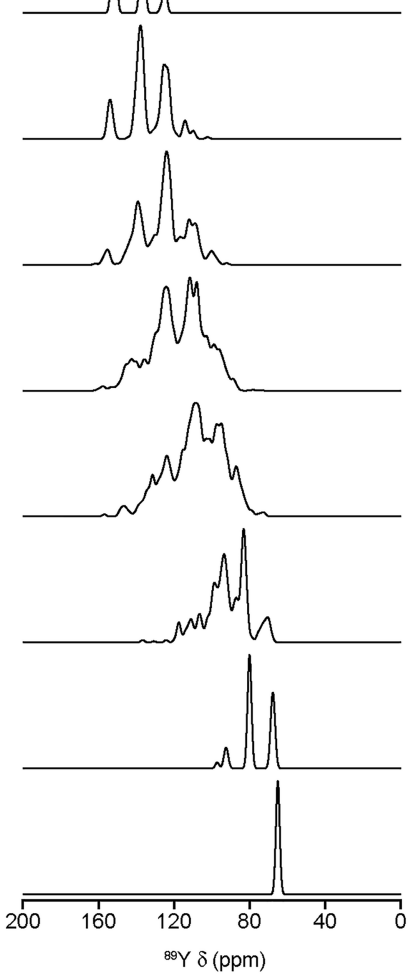

C
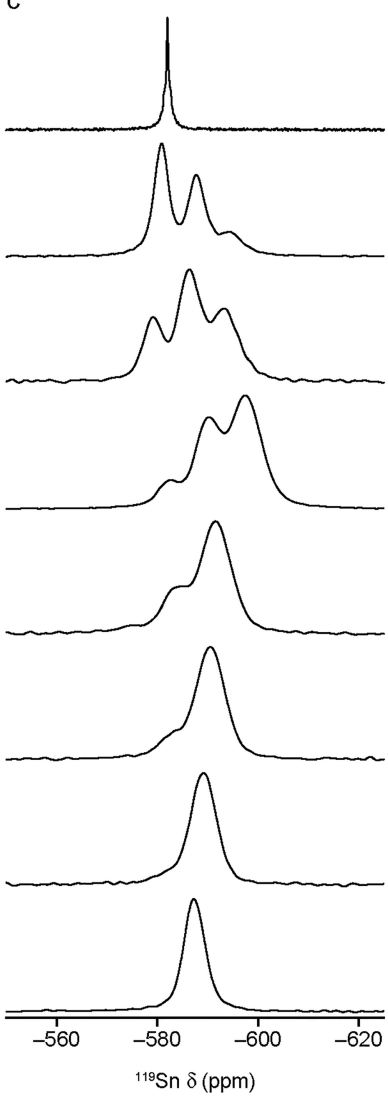

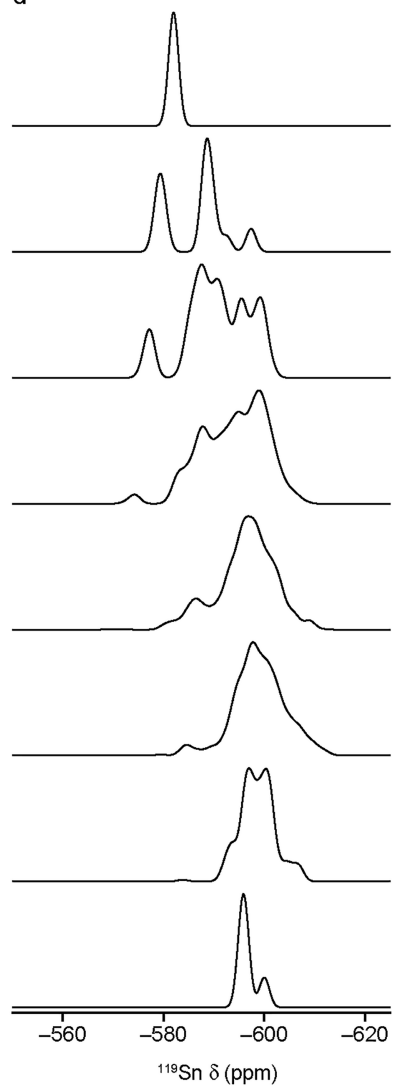

Figure 3. (a, c) Experimental and (b, d) predicted, assuming the limit of full disorder, (a, b) ${ }^{89} \mathrm{Y}$ and (c, d) ${ }^{119} \mathrm{Sn}$ MAS NMR spectra of $\mathrm{Y}_{2}\left(\mathrm{Sn}_{x} \mathrm{Ti}_{1-x}\right)_{2} \mathrm{O}_{7}$ for $x=0$ to 1 .

the spectral lineshapes. This also explains the observation in previous computational work (which modeled disorder simply by varying the number and arrangement of $\mathrm{Sn} / \mathrm{Ti}$ cations around just one $\mathrm{Y}$ (or $\mathrm{Sn}$ ) cation in the unit cell, in a simple "cluster-like" approach) of unusual values of $\delta_{\text {iso }}^{\text {calc }}$ for some species (i.e., values very different from those observed for similar local environments). ${ }^{13,14}$

It is clear from Figure $4 \mathrm{c}$ that when more structural models and variations in the longer-range environment are considered,

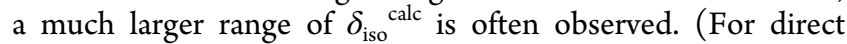
comparison, see the Supporting Information for calculations modeling disorder using this "cluster-like" approach, performed with the parameters and version of the code given in the Computational Methods.) Figure 5a reveals that these unusual shifts result from a deviation of the $\mathrm{O}_{8 \mathrm{a}}-\mathrm{Y}-\mathrm{O}_{8 \mathrm{a}}$ bond angle away from the value of $180^{\circ}$ observed for the ideal pyrochlore structure. However, there is little effect of this distortion (which can be quite significant, at up to $18^{\circ}$ in the most extreme cases) on ${ }^{89} \mathrm{Y} \Omega^{\text {calc }}$, as shown in Figure $5 \mathrm{~b}$. As shown in the Supporting Information, ${ }^{89} \mathrm{Y} \Omega^{\text {calc }}$ depends primarily on the number of Sn NNN, $n$, and on the mean Y$\mathrm{O}_{8 \mathrm{a}}$ distance, in agreement with previous work. ${ }^{16}$

The decomposition of the predicted ${ }^{119} \mathrm{Sn}$ MAS NMR spectrum of $\mathrm{Y}_{2} \mathrm{SnTiO}_{7}$ in Figure $4 \mathrm{~d}$ and e reveals a significant overlap of contributions from the different NNN arrangements of cations around Sn. As shown in the Supporting Information, a similar level of overlap is seen for most compositions. Figure 4f shows that there is a decrease in ${ }^{119} \mathrm{Sn} \delta_{\text {iso }}$ calc as $\mathrm{Ti}$ is substituted onto the NNN B sites, ${ }^{15}$ but that this decrease is smaller in magnitude as the number of Ti NNN increases. This results, as shown in Figure $3 \mathrm{c}$ and $\mathrm{d}$, in increased overlap of the spectral resonances as the level of $\mathrm{Ti}$ substitution increases, preventing any accurate decomposition of the ${ }^{119} \mathrm{Sn}$ spectral lineshape and hindering the extraction of detailed information about cation disorder. However, there is generally good agreement between the experimental and predicted spectra, validating the ensemble-based approach used.

The good agreement observed between experimental and predicted NMR spectra suggests there is an essentially random distribution of the $\mathrm{B}$ site cations in these materials; that is, the limit of full disorder, where individual subspectra are weighted only by the configurational degeneracy of the corresponding structural model, holds. For comparison, Figure 6 shows ${ }^{89} \mathrm{Y}$ NMR spectra of $\mathrm{Y}_{2} \mathrm{SnTiO}_{7}$ simulated with an additional Boltzmann weighting for each subspectrum, determined according to the relative energies of the structural models (as described in the Supporting Information). Spectra have been simulated for temperatures of 298, 500, 1000, and 2000 $\mathrm{K}$, although it should be noted that the earlier thermodynamic analysis predicts theoretically a miscibility gap would exist for this solid solution at lower temperature. At the lower temperatures, increased intensity is seen at the extremities of the spectral range, suggesting the structural models with lower relative energy have more $\mathrm{Sn}$-rich and Ti-rich local environ- 
a

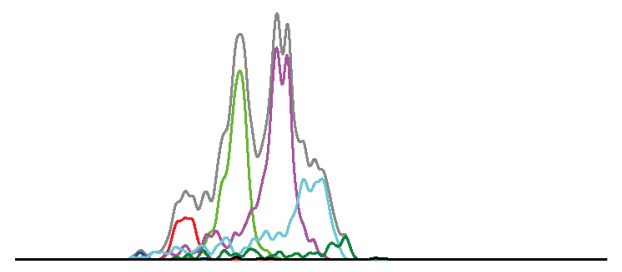

b
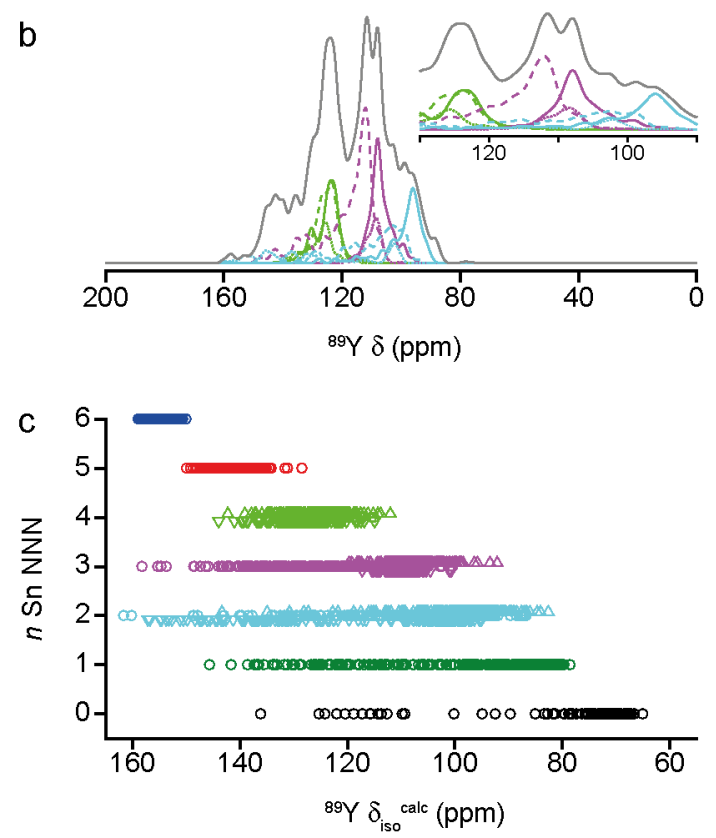

d

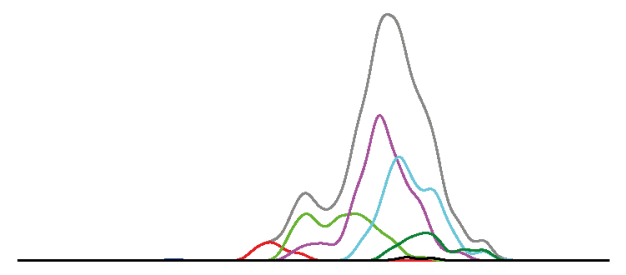

$-\mathrm{Sn} 6$

- Sn5Ti

- Sn4Ti2

- Sn3Ti3

- Sn2Ti4

- SnTi5

- Ti6

- Overall

e

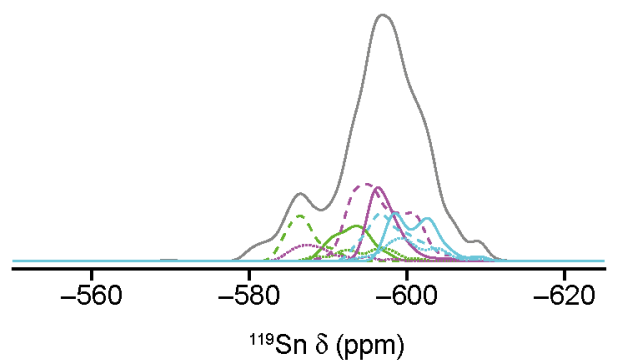

$-1,2-\mathrm{Sn} 4 \mathrm{Ti} 2$

$-1,3-\mathrm{Sn} 4 \mathrm{Ti} 2$

$-1,4-\mathrm{Sn} 4 \mathrm{Ti} 2$

$-1,2,3-\operatorname{Sn} 3 \mathrm{Ti} 3$

$\ldots 1,2,4-\operatorname{Sn} 3 T i 3$

$-1,3,5-\mathrm{Sn} 3 \mathrm{Ti} 3$

$-5,6-\mathrm{Sn} 2 \mathrm{Ti} 4$

4,6-Sn2Ti4

$-3,6-\mathrm{Sn} 2 \mathrm{Ti} 4$

- Overall

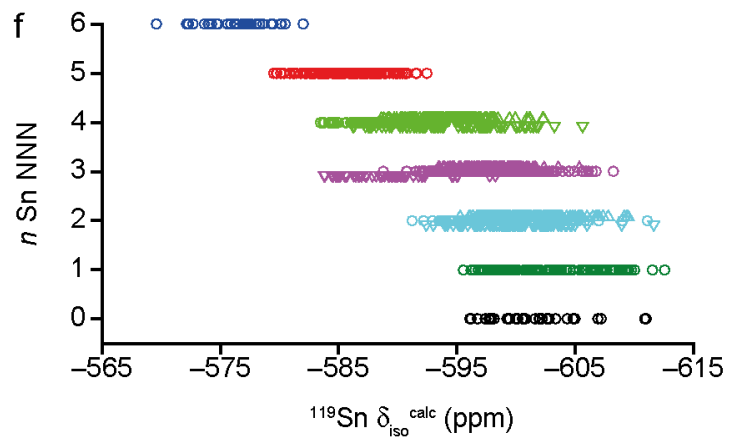

- Sn6

- Sn5Ti

$\triangle 1,2-S n 4 T i 2$

- 1,3-Sn4Ti2

$\nabla$ 1,4-Sn4Ti2

$\triangle 1,2,3-\mathrm{Sn} 3 \mathrm{Ti} 3$

- 1,2,4-Sn3Ti3

$\checkmark 1,3,5-\mathrm{Sn} 3 \mathrm{Ti} 3$

5.6-Sn2Ti4

4,6-Sn2Ti4

- SnTi5

- Ti6 $\checkmark 3,6-\mathrm{Sn} 2 \mathrm{Ti} 4$

Figure 4. Predicted, assuming the limit of full disorder, (a, b) ${ }^{89} \mathrm{Y}$ and $(\mathrm{d}, \mathrm{e}){ }^{119} \mathrm{Sn}$ MAS NMR spectra of $\mathrm{Y}_{2} \mathrm{SnTiO}_{7} .(\mathrm{c}, \mathrm{f}) \mathrm{Plots}$ of $(\mathrm{c}){ }^{89} \mathrm{Y}$ and $(\mathrm{f})$ ${ }^{119} \mathrm{Sn} \delta_{\text {iso }}$ calc as a function of the number of Sn NNN, $n$, for all Y and $\mathrm{Sn}$ species in all SOD-generated structural models.

a
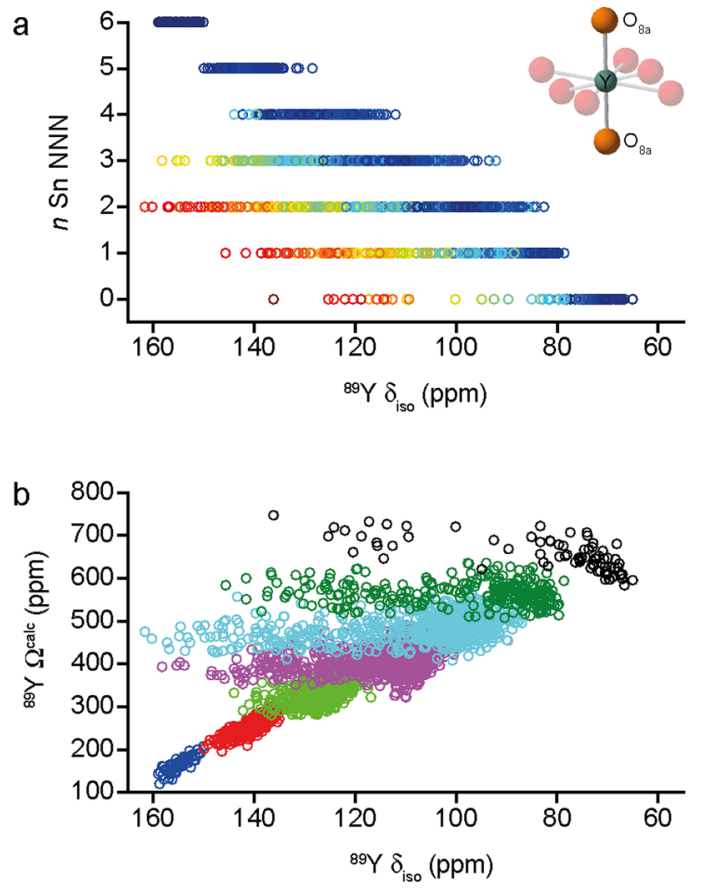

Figure 5. (a) Plot of ${ }^{89} \mathrm{Y} \delta_{\text {iso }}$ calc as a function of the number of $\mathrm{Sn}$ $\mathrm{NNN}, n$, for all $\mathrm{Y}$ and $\mathrm{Sn}$ species in all SOD-generated structural models of $\mathrm{Y}_{2}(\mathrm{SnTi})_{2} \mathrm{O}_{7}$, with points colored according to the deviation of the $\mathrm{O}_{8 \mathrm{a}}-\mathrm{Y}-\mathrm{O}_{8 \mathrm{a}}$ bond angle away from $180^{\circ}$. (b) Plot of ${ }^{89} \mathrm{Y} \delta_{\text {iso }}$ calc against $\Omega^{\text {calc }}$, with points colored according to $n$.
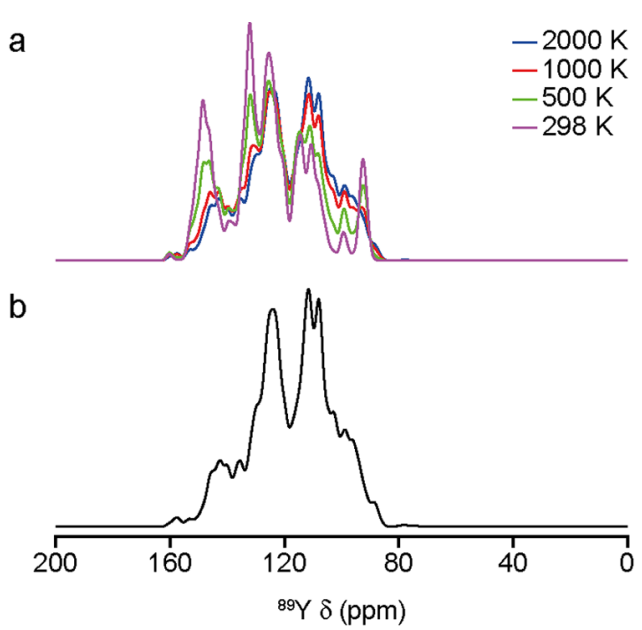

Figure 6. (a) Predicted ${ }^{89} \mathrm{Y}$ MAS NMR spectra of $\mathrm{Y}_{2} \mathrm{SnTiO}_{7}$ including a Boltzman weighting of the structural models for $T=298$, 500,1000 , and $2000 \mathrm{~K}$. For comparison, a similar plot in the limit of full disorder is shown in (b).

ments, i.e., that these models have more clustering of like cations. However, in general, better agreement with the experimental spectrum is seen at the higher temperatures, suggesting that the assumption of the limit of full disorder is reasonable in this system and likely reflects the higher synthesis temperatures used experimentally. It is clear from this analysis that no configurational equilibrium is established at lower temperatures, which is consistent with the prediction above 
that thermodynamic equilibrium at low temperatures involves phase separation. Movies included in the Supporting Information also show that each simulated $\mathrm{Y}_{2} \mathrm{SnTiO}_{7}$ subspectrum is very different, but that only when a large number of these are combined does the spectrum approach that seen experimentally.

It is known that some properties of solid solutions can be evaluated using a single configuration (or SQS, as described above) that best represents the mixed system. ${ }^{10}$ For $\mathrm{Y}_{2} \mathrm{SnTiO}_{7}$, the structural model generated by SOD that is closest to the SQS model has an energy close to that of the ensemble average for this composition and exhibits average numbers of first- and second-nearest neighbor $\mathrm{Ti}-\mathrm{Ti}, \mathrm{Sn}-\mathrm{Ti}$, and $\mathrm{Sn}-\mathrm{Sn}$ pairs identical to the perfectly disordered structure for this composition. The ${ }^{89} \mathrm{Y}$ MAS NMR spectrum simulated for this structural model is shown in the Supporting Information. Although this spectrum has better agreement with experiment than those for most structural models for this composition (see S6.1 in the Supporting Information), it is clear that it is not possible to reproduce either the summed simulated spectrum (i.e., the full disorder limit) or the experimental NMR spectrum with one "average" structure, illustrating the sensitivity of the NMR parameters to the specific local environment.

Although there is generally good agreement between experimental and simulated spectra, Figure 3 shows that for both ${ }^{89} \mathrm{Y}$ and ${ }^{119} \mathrm{Sn} \mathrm{NMR}$, the agreement is poorer at lower levels of $\mathrm{Ti} / \mathrm{Sn}$ substitution. For example, when $x=0.875$ the number and position of the peaks seen in the simulated ${ }^{89} \mathrm{Y}$ NMR spectra match very well with experiment, but the relative intensities are in poorer agreement, with a Sn6:Sn5Ti:Sn4Ti4 ratio of 1.0:0.7:0.4 seen experimentally and 1.00:1.33:0.33 in the simulated spectrum, as shown in Table 2. For this example,

Table 2. Probability of Finding Y Atoms with $\boldsymbol{n}$ Sn NNN in Different Structural Models of $\mathrm{Y}_{2}\left(\mathrm{Sn}_{0.875} \mathrm{Ti}_{0.125}\right)_{2} \mathrm{O}_{7}{ }^{a}$

\begin{tabular}{|ccccc}
$\begin{array}{c}\text { NNN } \\
\text { environment }\end{array}$ & unit cell & $\begin{array}{c}1 \times 1 \times 2 \\
\text { supercell }\end{array}$ & infinite cell & experiment \\
\multicolumn{1}{c}{ Sn6 } & 0.3750 & 0.4157 & 0.4488 & $0.48(5)$ \\
Sn5Ti & 0.5000 & 0.4338 & 0.3847 & $0.33(5)$ \\
Sn4Ti2 & 0.1250 & 0.1356 & 0.1374 & $0.19(5)$ \\
Sn3Ti3 & 0.0000 & 0.0145 & 0.0262 & \\
Sn2Ti4 & 0.0000 & 0.0004 & 0.0028 & \\
SnTi5 & 0.0000 & 0.0000 & 0.0002 & \\
Ti6 & 0.0000 & 0.0000 & $4 \times 10^{-6}$ &
\end{tabular}

${ }^{a_{T}}$ The corresponding data from experiment (Figure $3 \mathrm{a}, x=0.875$ ) are shown for comparison.

this difference cannot arise from the assumption of full disorder or the need to apply any Boltzmann weighting; only three unique arrangements of atoms are possible within the unit cell for this composition (with $g_{\mathrm{m}}=48,24$, and 48), but all have the same number of Y species with 6, 5, and 4 Sn NNN. While applying a Boltzmann weighting might have small effects on the intensities at an exact chemical shift (and therefore the width and shape of the lines in the simulated spectrum), it would not affect the integrated spectral intensities. It is clear that to achieve any better agreement with experiment at these low concentrations it would be necessary to consider disorder on a length scale greater than the unit cell, where all the possible local and longer-range environments are represented. The use of a supercell, however, would be extremely computationally demanding at this level of theory, with even a $1 \times 1 \times 2$ supercell having 346 possible unique arrangements of atoms (35960 total arrangements) for $x=0.875$. The probability of finding $\mathrm{Y}$ atoms with $n \mathrm{Sn} \mathrm{NNN}$ for this extended supercell (taking into account the degeneracies of each structural model) is given in Table 2 . These are closer to the relative intensities seen in the experimental spectrum. However, the number of structural models that need to be considered increases significantly when the level of substitution increases, and with the size of the supercell, ensemble modeling and computation quickly become unfeasible.

It is possible to predict the probability of finding $\mathrm{Y}$ atoms with $n$ Sn NNN in the limit of an infinite supercell (assuming random disorder) using simple statistics. According to the binomial theorem, the probability $(P)$ of a Y atom having $n$ Sn $\mathrm{NNN}$ is given by

$$
P(n \mathrm{Sn} \mathrm{NNN})=\Omega x^{n}(1-x)^{6-n}
$$

where $\Omega$ is the number of possible permutations of the $n$ substitutions on the six surrounding B sites and $x$ denotes the chemical composition. The improved agreement with experiment (seen in Table 2) suggests that disorder is relevant on length scales greater than that of the unit cell. However, such a simple statistical approach is only possible in the limit of full disorder, as a Boltzmann weighting cannot be applied to individual atomic arrangements. Furthermore, comparison to experiment is only possible when well-resolved resonances, which can be easily attributed to a single and specific local environment, can be identified. While this is the case for the ${ }^{89} \mathrm{Y}$ NMR spectrum of $\mathrm{Y}_{2}\left(\mathrm{Sn}_{x} \mathrm{Ti}_{1-x}\right)_{2} \mathrm{O}_{7}$ when $x=0.875$, as the $\mathrm{Ti}$ content increases, the spectral lineshapes become more complex and more overlapped (as shown in Figure 3), preventing such a simple analysis and comparison. It is also worth noting, for example, that although Figure 6 shows generally better agreement is obtained between experiment and theory at higher temperatures for $\mathrm{Y}_{2} \mathrm{SnTiO}_{7}$, this does result in poorer agreement at the highest shifts. While this could indicate some weak preference for clustering or ordering, these differences could also result from the limitations of using a single unit cell. When the spectral overlap is significant, the ensemble approach (even for a single unit cell) is crucial for understanding the contributions to the spectrum, the origin of the spectral lineshapes observed, and the relative importance of different atomic arrangements in the real material. More sophisticated approaches need to be developed that are accurate at both low and intermediate concentrations, possibly based on grand-canonical ensembles where shifts obtained for supercells with different compositions could be combined to model the NMR spectrum at one composition.

\section{- CONCLUSIONS}

While disorder can have a significant impact on the physical and chemical properties of a material, the characterization of disordered solids poses a considerable challenge, for both experiment and computation. The sensitivity of NMR spectroscopy to the atomic-scale environment provides an ideal opportunity for understanding disorder at the local level, rather than relying on a structural picture averaged over space and time. Here, we have shown that ensemble-based modeling can be used to interpret the complex and overlapped spectral lineshapes than often result in disordered materials, providing insight into the structural models likely to be relevant to the 
real system. Conventionally, in NMR spectroscopy considerable effort is focused on the assignment of signals to specific types of chemical environments. However, it is clear from the results shown here that this is not always possible for complex and disordered solids, with considerable variation in the shifts seen for similar environments and significant overlap of signals resulting from different environments. Indeed, although it is tempting to use this computational work to facilitate spectral assignment, this is, in fact, nonsensical, as the driving force for spectral assignment is to obtain an understanding of the type (and proportion) of species present in order to construct structural models that are consistent with these observations. However, a complete set of structural models is available directly in the ensemble-based modeling approach exploited here, with DFT enabling insight into their relative energies and the NMR parameters associated with different species. Structural insight is obtained by direct comparison to the experimental spectrum, rather than in a two-step process that relies initially on accurate spectral interpretation and analysis. The possession of a complete set of structural models for a system is, however, able to provide an understanding of the origin of the interactions that affect the nuclear spins and, in particular, their dependence on geometric parameters. This fundamental insight will be useful for complex or larger systems, where it may not be possible to generate a complete set of models.

For $\mathrm{Y}_{2}(\mathrm{Sn}, \mathrm{Ti})_{2} \mathrm{O}_{7}$ we have shown good agreement between predicted and experimental spectra in the limit of full disorder, i.e., where the summed spectrum for a fixed composition contains subspectra that are weighted only by their configurational degeneracy. The inclusion of a Boltzmann weighting confirms best agreement with experiment is obtained for higher temperatures, but highlights that the atomic configurations with lower enthalpy contain more clustering of like atoms. It is also interesting to note that the thermodynamic analysis (using the subregular solid solution model) predicts a miscibility gap in this system at temperatures of $<800 \mathrm{~K}$, with the observation of a solid solution experimentally suggesting the materials produced are metastable at room temperature: the high-temperature cation distribution is frozen and diffusion barriers prevent configurational equilibrium at lower temperatures. This analysis also reveals that it is energetically less favorable to substitute the smaller $\mathrm{Ti}^{4+}$ cation into $\mathrm{Y}_{2} \mathrm{Sn}_{2} \mathrm{O}_{7}$ than vice versa, initially perhaps a surprising observation. However, although the $\mathrm{Y}_{2} \mathrm{Ti}_{2} \mathrm{O}_{7}$ pyrochlore itself is stable, $\mathrm{Ti}^{4+}$ appears too small to satisfy the bonding requirements of the surrounding oxygens in the Sn-rich material owing to the more covalent nature of the $\mathrm{Sn}-\mathrm{O}$ bond, which leads to a less flexible structure, as discussed in the Supporting Information.

Although the experimental and predicted spectra show a high level of similarity, the intensity of the spectral resonances is in poorer agreement at low levels of substitution. The restricted number of unique structural models in these cases suggests that disorder should be considered at a length scale greater than that of the ordered unit cell to achieve better agreement with experiment. Although currently computationally demanding in many cases (particularly for the subsequent calculation of NMR parameters), more routine use of supercells may become possible with future advances in hardware or could be possible in cases where there is less significant overlap of the spectral lineshapes and analysis need only consider the number of each type of environment present. As the level of disorder increases, it will, of course, become impossible to generate a "complete set" of structures, and alternative approaches will be required to generate sufficiently large sets of chemically relevant structural models. The approach previously used for glasses, where models were generated using molecular dynamics, ${ }^{4,33}$ will become increasingly useful in these cases, as will the methods emerging from new machine learning work. ${ }^{34}$

In conclusion, we have shown that ensemble-based modeling approaches offer great potential for understanding configurational disorder, especially when used in conjunction with experimental NMR spectroscopy. Such an approach does, however, require a change in the mindset of the spectroscopist, moving away from the need for a detailed understanding of every resonance, shoulder or splitting observed in the experimental spectrum, but instead viewing the whole spectrum as the combined result of signals from a set of structural models that can be directly compared to computational predictions. With future developments in hardware and in computational methods, ensemble-based approaches for larger and more complicated systems will become possible, enabling more detailed structural insight and aiding future materials design.

\section{ASSOCIATED CONTENT}

\section{S Supporting Information}

The Supporting Information is available free of charge on the ACS Publications website at DOI: 10.1021/jacs.9b09036.

Information on convergence and referencing of DFT calculations, calculation of thermodynamic properties, decomposition of spectral lineshapes, calculations using "cluster-based" models, information on ${ }^{89} \mathrm{Y}$ shielding anisotropy. and simulated subspectra for individual models (PDF)

Supporting movie (MP4)

(MP4)

\section{AUTHOR INFORMATION}

\section{Corresponding Authors}

*r.grau-crespo@reading.ac.uk

*sema@st-andrews.ac.uk

ORCID ${ }^{\circ}$

David McKay: 0000-0003-0362-7848

Ricardo Grau-Crespo: 0000-0001-8845-1719

Sharon E. Ashbrook: 0000-0002-4538-6782

Notes

The authors declare no competing financial interest.

\section{ACKNOWLEDGMENTS}

The authors would like to thank the ERC (EU FP7 Consolidator Grant 614290 “EXONMR”). S.E.A. would like to thank the Royal Society and Wolfson Foundation for a merit award. We acknowledge support from the Collaborative Computational Project on NMR Crystallography CCP-NC funded by EPSRC (EP/M022501/1) and the UKCP consortium funded by EPSRC (EP/K013564/1). For computational resources we are grateful to the UK Materials and Molecular Modelling Hub, which is partially funded by EPSRC (EP/P020194/1), and to the UK HPC Materials Chemistry Consortium, which is funded by EPSRC (EP/ L000202). The research data (and/or materials) supporting this publication can be accessed in refs 32 and 35 . 


\section{REFERENCES}

(1) Capasso, F. Band-Gap Engineering: From Physics and Materials to New Semiconductor Devices. Science 1987, 235, 172-176.

(2) Ashbrook, S. E.; Dawson, D. M.; Griffin, J. M. Solid-State Nuclear Magnetic Resonance Spectroscopy. In Local Structure Characterisation; Bruce, D. W., O’Hare, D., Walton, R. I., Eds.; John Wiley \& Sons Ltd, 2014; pp 1-88.

(3) Moran, R. F.; Dawson, D. M.; Ashbrook, S. E. Exploiting NMR Spectroscopy for the Study of Disorder in Solids. Int. Rev. Phys. Chem. 2017, 35, 39-115.

(4) Bonhomme, C.; Gervais, C.; Babonneau, F.; Coelho, C.; Pourpoint, F.; Azais, T.; Ashbrook, S. E.; Griffin, J. M.; Yates, J. R.; Mauri, F.; Pickard, C. J. First-Principles Calculation of NMR Parameters Using the Gauge Including Projector Augmented Wave Method: A Chemist's Point of View. Chem. Rev. 2012, 112, 57335779.

(5) Ashbrook, S. E.; McKay, D. Combining Solid-State NMR Spectroscopy with First-Principles Calculations - a Guide to NMR Crystallography. Chem. Commun. 2016, 52, 7186-7204.

(6) Grau-Crespo, R.; Waghmare, U. V. Simulation of Crystals with Chemical Disorder at Lattice Sites. In Molecular Modeling for the Design of Novel Performance Chemicals and Materials; Rai, B., Ed.; CRC Press, 2012; pp 299-322.

(7) Sanchez, J. M.; Ducastelle, F.; Gratias, D. Generalized Cluster Description of Multicomponent Systems. Phys. A 1984, 128, 334350.

(8) Wu, Q.; Bong, H.; Song, T.; Gao, J.; Shi, S. Cluster Expansion Method and its Application in Computational Materials Science. Comput. Mater. Sci. 2016, 125, 243-254.

(9) Zunger, A.; Wang, L. G.; Hart, G. L. W.; Sanati, M. Obtaining Ising-like Expansions for Binary Alloys from First Principles. Modelling Simul. Mater. Sci. Eng. 2002, 10, 685-706.

(10) Zunger, A.; Wei, S. H.; Ferreira, L. G.; Bernard, J. E. Special Quasirandom Structures. Phys. Rev. Lett. 1990, 65, 353-356.

(11) Grey, C. P.; Dobson, C. M.; Cheetham, A. K.; Jakeman, R. J. B. Studies of Rare-Earth Stannates by ${ }^{119} \mathrm{Sn}$ MAS NMR. The Use of Paramagnetic Shift Probes in the Solid State. J. Am. Chem. Soc. 1989, $111,505-511$.

(12) Grey, C. P.; Smith, M. E.; Cheetham, A. K.; Dobson, C. M.; Dupree, R. ${ }^{89} \mathrm{Y}$ MAS NMR Study of Rare-Earth Pyrochlores: Paramagnetic Shifts in the Solid State. J. Am. Chem. Soc. 1990, 112, 4670-4765.

(13) Ashbrook, S. E.; Whittle, K. R.; Lumpkin, G. R.; Farnan, I. ${ }^{89} \mathrm{Y}$ Magic-Angle Spinning NMR of $\mathrm{Y}_{2} \mathrm{Ti}_{2-x} \mathrm{Sn}_{x} \mathrm{O}_{7}$ Pyrochlores. J. Phys. Chem. B 2006, 110, 10358-10364.

(14) Reader, S. W.; Mitchell, M. R.; Johnston, K. E.; Pickard, C. J.; Whittle, K. R.; Ashbrook, S. E. Cation Disorder in Pyrochlore Ceramics: ${ }^{89}$ Y MAS NMR and First-Principles Calculations. J. Phys. Chem. C 2009, 113, 18874-18883.

(15) Mitchell, M. R.; Reader, S. W.; Johnston, K. E.; Pickard, C. J.; Whittle, K. R.; Ashbrook, S. E. ${ }^{119}$ Sn MAS NMR and First-Principles Calculations for the Investigation of Disorder in Stannate Pyrochlores. Phys. Chem. Chem. Phys. 2011, 13, 488-497.

(16) Mitchell, M. R.; Carnevale, D.; Orr, R.; Whittle, K. R.; Ashbrook, S. E. Exploiting the Chemical Shielding Anisotropy to Probe Structure and Disorder in Ceramics: ${ }^{89} \mathrm{Y}$ MAS NMR and FirstPrinciples Calculations. J. Phys. Chem. C 2012, 116, 4273-4286.

(17) Fernandes, A.; Moran, R. F.; Sneddon, S.; Dawson, D. M.; McKay, D.; Bignami, G. P. M.; Blanc, F.; Whittle, K. R.; Ashbrook, S. E. ${ }^{17} \mathrm{O}$ Solid-State NMR Spectroscopy of $\mathrm{A}_{2} \mathrm{~B}_{2} \mathrm{O}_{7}$ Oxides: Quantitative Isotopic Enrichment and Spectral Acquisition? RSC Adv. 2018, 8, 7089-7101.

(18) Grau-Crespo, R.; Hamad, S.; Catlow, C. R. A.; de Leeuw, N. H. Symmetry-Adapted Configurational Modelling of Fractional Site Occupancy in Solids. J. Phys.: Condens. Matter 2007, 19, 256201.

(19) Pickard, C. J.; Mauri, F. All-Electron Magnetic Response with Pseudopotentials: NMR Chemical Shifts. Phys. Rev. B: Condens. Matter Mater. Phys. 2001, 63, 245101.
(20) Clark, S. J.; Segall, M. D.; Pickard, C. J.; Hasnip, P. J.; Probert, M. J.; Refson, K.; Payne, M. C. First-Principles Methods using CASTEP. Z. Kristallogr. - Cryst. Mater. 2005, 220, 567-570.

(21) Perdew, J. P.; Burke, K.; Ernzerhof, M. Generalized Gradient Approximation Made Simple. Phys. Rev. Lett. 1996, 77, 1865-1868.

(22) Vanderbilt, D. Soft Self-Consistent Pseudopotentials in a Generalized Eigenvalue Formalism. Phys. Rev. B: Condens. Matter Mater. Phys. 1990, 41, 7892-7895.

(23) Yates, J. R.; Pickard, C. J.; Payne, M. C.; Mauri, F. Relativistic Nuclear Magnetic Resonance Chemical Shifts of Heavy Nuclei with Pseudopotentials and the Zeroth-Order Regular Approximation. J. Chem. Phys. 2003, 118, 5746-5743.

(24) Monkhorst, H. J.; Pack, J. D. Special Points for Brillouin-Zone Integrations. Phys. Rev. B 1976, 13, 5188-5192.

(25) Ganguly, J. Thermodynamics in Earth and Planetary Sciences; Springer-Verlag: Berlin, 2008.

(26) Kennedy, B. J. Structural Trends in Pyrochlore Oxides. Mater. Sci. Forum 1996, 228, 753.

(27) Subramanian, M. A.; Aravamudan, G.; Subba Rao, G. V. Oxide Pyrochlores - a Review. Prog. Solid State Chem. 1983, 15, 55-143.

(28) Chakoumakos, B. C. Systematics of the Pyrochlore Structure Type, Ideal $\mathrm{A}_{2} \mathrm{~B}_{2} \mathrm{X}_{6} \mathrm{Y}$. J. Solid State Chem. 1984, 53, 120-129.

(29) Shannon, R. D. Revised Effective Ionic Radii and Systematic Studies of Interatomie Distances in Halides and Chaleogenides. Acta Crystallogr., Sect. A: Cryst. Phys., Diffr., Theor. Gen. Crystallogr. 1976, A32, 751-767.

(30) Benny, S.; Grau-Crespo, R.; de Leeuw, N. A Theoretical Investigation of $\alpha-\mathrm{Fe}_{2} \mathrm{O}_{3}-\mathrm{Cr}_{2} \mathrm{O}_{3}$ Solid Solutions. Phys. Chem. Chem. Phys. 2009, 11, 808-815.

(31) Ektarawong, A.; Simak, S. I.; Alling, B. First-Principles Prediction of Stabilities and Instabilities of Compounds and Alloys in the Ternary B-As-P System. Phys. Rev. B: Condens. Matter Mater. Phys. 2017, 96, 024202.

(32) Moran, R. F. Investigating the Use of First-Principles Calculations for NMR Studies of Disorder in the Solid State, Ph.D. Thesis, University of St Andrews, 2019.

(33) Charpentier, T.; Menziani, M. C.; Pedone, A. Computational Simulations of Solid State NMR spectra: A New Era in Structure Determination of Oxide Glasses. RSC Adv. 2013, 3, 10550-10578.

(34) Chaker, Z.; Salanne, M.; Delaye, J. M.; Charpentier, T. NMR Shifts in Aluminosilicate Glasses via Machine Learning. Phys. Chem. Chem. Phys. 2019, 21, 21709-21725.

(35) Moran, R. F.; McKay, D.; Tornstrom, P. C.; Aziz, A.; Fernandes, A.; Grau-Crespo, R.; Ashbrook, S. E. Ensemble-Based Modelling of the NMR Spectra of Solid Solutions: Cation Disorder in $\mathrm{Y}_{2}(\mathrm{Sn}, \mathrm{Ti})_{2} \mathrm{O}_{7}$. Dataset; University of St Andrews Research Portal, DOI: 10.17630/47d302a7-1119-47ad-9db3-effe4d3d3bee, 2019. 